\title{
La caja de herramientas del profesor: Un portafolio construido de manera colaborativa.
}

\author{
José M. ${ }^{a}$ Falcó Boudet ${ }^{\mathrm{a}}$, Miguel García Garcés ${ }^{\mathrm{b}}$ y José Luis Huertas Talón ${ }^{\mathrm{b}}$
}

${ }^{a}$ Dpto. Informática e Ingeniería de Sistemas (Facultad de Educación, Universidad de Zaragoza) chema.falco@unizar.es, 'b Dto. de Ingeniería de Diseño y Fabricación (Escuela de Ingeniería y Arquitectura, Universidad de Zaragoza)mggarces@unizar.es y jhuertas@unizar.es.

\begin{abstract}
This experience aimed to bring what students of specialties of Technology and Computers of a teachers' training course have learned in the classroom to the reality of their professional future. The main objective was that future teachers developed skills necessary to teachers by building the whole group class a Teacher's Toolbox. Each student has designed a tool weekly following a specific set of rules. A wiki has been used as support for the toolbox, which has facilitated collaboration among all students. To assess the experience teachers have used an oline questionnaire and have analyzed the tools created. Questionnaire findings confirm that students value the toolbox as a means of learning and also they evaluate as positive teacher and peers feedback. Tools' analysis shows that students have learned to design appropriate learning activities to achieve goals set.
\end{abstract}

Keywords: Teacher's toolbox, Portfolio, Collaborative learning, Teacher training

\section{Resumen}

Esta experiencia buscaba acercar lo aprendido en el aula por los estudiantes de las especialidades de Tecnología e Informática de Secundaria del Máster en profesorado de la Universidad de Zaragoza a la realidad de su futuro profesional. El objetivo principal era que los futuros docentes desarrollaran habilidades necesarias para ejercer como tales al tiempo que construían entre todo el grupo de clase una "Caja de herramientas del profesor". El procedimiento seguido ha sido la elaboración semanal por cada estudiante de una herramienta de acuerdo a las características especificadas. Como soporte para la caja de herramientas se ha utilizado una wiki que ha facilitado la colaboración entre todos los estudiantes. Para valorar los resultados se han utilizado una encuesta y el análisis de las herramientas creadas. Los resultados de la encuesta indican que los estudiantes valoran la caja de herramientas como medio de aprendizaje y que también valoran positivamente la retroalimentación proporcionada por el profesor y por sus compañeros. El análisis de las herramientas demuestra que los estudiantes han aprendido a diseñar actividades de aprendizaje adecuadas al grupo de alumnos y a los objetivos establecidos.
} 
Palabras clave: caja de herramientas, portafolio, aprendizaje colaborativo, máster en profesorado.

\section{Introducción}

Toda profesión tiene un conjunto de herramientas básicas necesarias para desempeñar su trabajo. Sin embargo, la enseñanza se ha percibido como una profesión que solo requería de una pizarra, tiza y alumnos. Sin entrar en la discusión de si esto es cierto o no, lo que si resulta evidente es que en el proceso de enseñanza - aprendizaje del s. XXI es necesario el empleo de las Tecnologías de la Información y la Comunicación (TIC) para que la enseñanza sea más efectiva y el aprendizaje menos aburrido.

Los alumnos del Máster Universitario en Profesorado de Educación Secundaria Obligatoria, Bachillerato, Formación Profesional y Enseñanzas de Idiomas, Artísticas y Deportivas de la Universidad de Zaragoza deben ser, de acuerdo con el modelo TPACK (Koehler y Mishra, 2008), profesionales formados para integrar en su práctica docente tanto la didáctica como las TIC con los conocimientos propios de su disciplina.

No se trata de que utilicen las TIC solo como herramienta de comunicación, ni de que sean meros consumidores de tecnología o que las utilizan más como fin que como medio, sino de que aprendan cuándo y cómo usarlas de una manera efectiva en su faceta de gestión del trabajo personal y en su faceta pedagógica, como herramientas para crear, colaborar, pensar críticamente y compartir. Esto requiere partir de la tarea o actividad de aprendizaje y reflexionar sobre cuál o cuáles son las herramientas más adecuadas, tecnológicas o no, para alcanzar los objetivos y no al revés. Si solo se presta atención a lo vistoso de una aplicación el aprendizaje logrado por los alumnos puede ser superficial. Una herramienta no puede distraernos del objetivo de aprendizaje que queremos lograr. "Quien compra un taladro no quiere una taladro, lo que quiere es un agujero” (Perry Marshall, 2014).

Durante el curso 2015-16 se propuso a los alumnos de la asignatura de Diseño, organización y desarrollo de actividades para el aprendizaje de Informática y Tecnología la elaboración de manera colectiva de una "Caja de herramientas del profesor".

En cursos anteriores los estudiantes de esta asignatura han construido portafolios individuales donde reflexionaban sobre sus aprendizajes y proponían actividades en los que ponerlos en práctica (Falcó, 2014). Esta experiencia cambia y amplia la perspectiva de ese portafolio. Por un lado, se construye un portafolio colectivo con la suma de las aportaciones individuales y las valoraciones de las aportaciones del resto del grupo. Por otro, se profundiza en la aplicación práctica en el ejercicio de la profesión, describiendo con más detalle la actividad propuesta.

Esta experiencia ha sido aprobada en el marco del Programa de Incentivación de la Innovación Docente de la Universidad de Zaragoza (PIIDUZ_15_237), dentro de la convocatoria de Innovación Docente 2015-2016. 


\section{La caja de herramientas. Concepto}

Una "Caja de herramientas del profesor" es eso, una caja de herramientas, un conjunto de actividades, proyectos, rúbricas y otros recursos al que pueden acudir tanto profesores noveles como veteranos para diseñar las sesiones de trabajo en el aula con sus alumnos.

No se trataba de 'coleccionar' un álbum de TIC's, sino de construir una "Caja de herramientas" sólida a la que poder recurrir en la práctica profesional. Distintos alumnos, distintos contextos o distintas fases del curso requieren de herramientas distintas. Igual que un martillo en si no es buena o mala herramienta, si no que es útil o no según la situación, una actividad de aprendizaje debe ser adecuada a la situación. Al diseñar la "Caja de herramientas" los estudiantes, cuya portada vemos en la figura 1, debían incluir una reflexión sobre lo adecuado de la TIC seleccionada y la actividad diseñada para lograr los objetivos marcados. Una herramienta será más adecuada para determinados proyectos que otra y otras serán más adecuadas para ciertas edades.

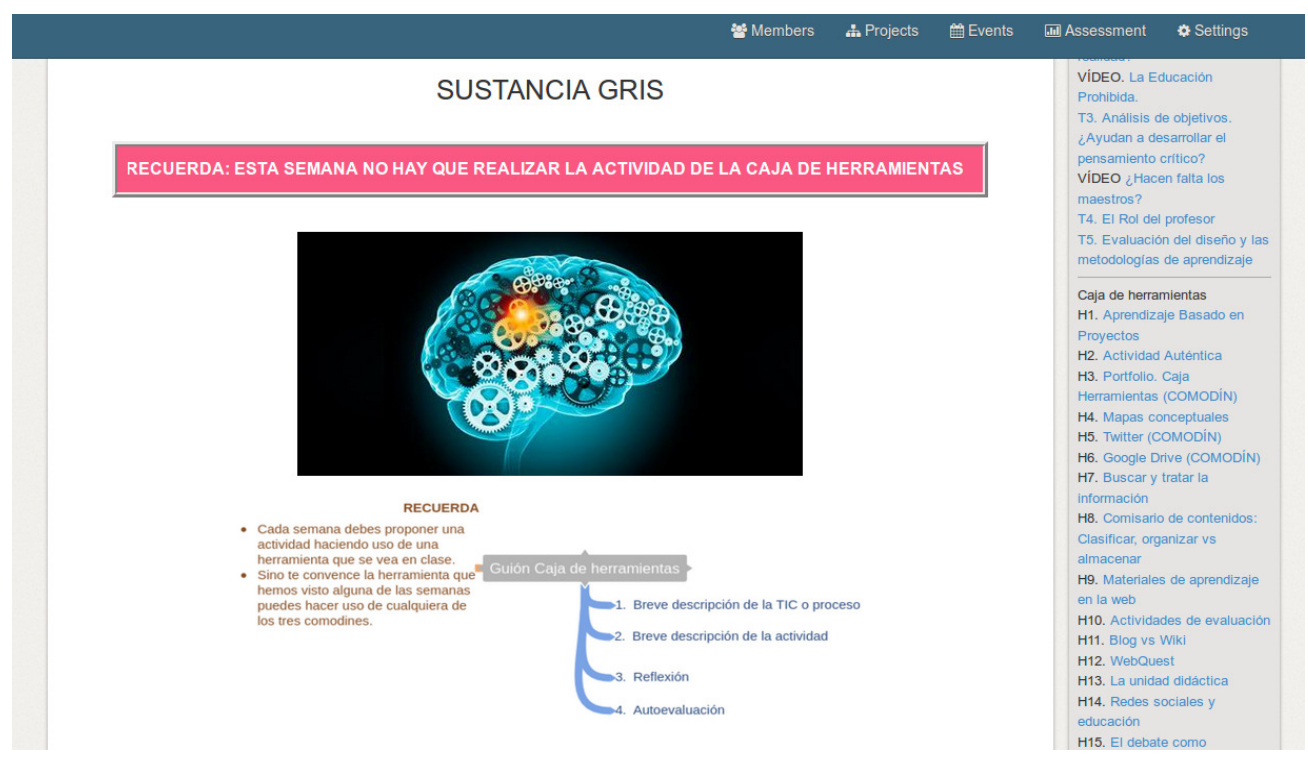

Fig 1: Portada de Sustancia Gris (la caja de herramientas)

Las cajas de herramientas varían de un profesional a otro. Construir la "Caja de herramientas del profesor" de manera colaborativa propicia que lo que cada estudiante ha aportado de manera individual permita al grupo aprender para su futuro desempeño profesional (Hanson, 2011), lo que no minimiza el reto de desarrollar personalmente una buena actividad de aprendizaje, sino más bien al contrario.

\section{Objetivos}

Esta experiencia tiene dos objetivos generales, uno en cuanto a portafolio de aprendizaje y otro en cuanto a la formación profesional que los estudiantes esperan. 
El objetivo de los profesores de la asignatura es que los estudiantes aprendan a construir herramientas e instrumentos para enriquecer y mejorar los procesos de enseñanza aprendizaje de sus futuros alumnos. Construir un portafolio colectivo, la "Caja de herramientas", ayuda a que reflexionen sobre su aprendizaje personal y a que lo hagan visible mediante el diseño y creación de un banco de herramientas del profesor y propicia el aprendizaje entre iguales.

El objetivo de los estudiantes del máster en profesorado es adquirir las competencias necesarias para el desempeño de la práctica profesional. Construir la "Caja de herramientas del profesor" les ayuda a desarrollarlas a tres niveles:

- Cada herramienta creada por un estudiante es reflejo de su estilo personal, de la selección y adaptación de los aprendizajes de la asignatura a su manera de ser profesor, le permite profundizar en sus motivaciones para ejercer esta profesión y explorar nuevas ideas.

- La elaboración colectiva de la "Caja de herramientas del profesor" desarrolla la percepción de que la docencia es un trabajo en equipo y permite compartir ideas y recursos.

- La "Caja de herramientas del profesor" es el inicio de su formación permanente como docente. La caja iniciada en este máster podrá ir aumentando y mejorando con las experiencias como profesores de los actuales y futuros estudiantes.

\section{Desarrollo de la innovación}

Como ha quedado indicado, esta experiencia se ha llevado a cabo durante el curso 2015-16 con los alumnos de la asignatura de Diseño, organización y desarrollo de actividades para el aprendizaje de Informática y Tecnología. La asignatura, de ocho créditos, está dividida en dos áreas, informática y tecnología, con contenidos separados. De los 24 alumnos matriculados $17(70,8 \%)$ participaron regularmente en la actividad.

\subsection{Actividades}

Cada estudiante debía completar una entrada semanal en el área de informática, proponiendo una herramienta de aprendizaje de alguna unidad de Tecnologías de Secundaria o Bachillerato basada en TIC, una valoración semanal de una de las actividades de otros compañeros de clase, valorando lo adecuado a los objetivos propuestos y la aplicabilidad de la actividad analizada, y, en paralelo, cada grupo de trabajo debía realizar un diario del proyecto construido en el área de tecnología.

Una herramienta de aprendizaje debía incluir:

a) descripción de la herramienta TIC a utilizar,

b) descripción de la actividad, incluyendo los objetivos de aprendizaje que se pretenden lograr con la misma, 
c) reflexión sobre la adecuación de esa actividad y esa TIC para lograr los objetivos propuestos

d) y autoevaluación de los aprendizajes que necesitaría el estudiante autor/a para llevarla a cabo con eficacia.

En la primera sección, descripción de la TIC elegida, el estudiante debía mostrar su conocimiento de la herramienta seleccionada. En la segunda debía diseñar una actividad con unos objetivos de aprendizaje bien definidos cuya realización requiriera el uso de la TIC descrita. En la tercera el estudiante utilizaba el pensamiento crítico para valorar si la actividad propuesta y la TIC elegida eran adecuadas para lograr los objetivos planteados con alumnos de las edades del curso para el que estaba diseñada. En la última sección, autoevaluación, el estudiante debía evaluar sus necesidades de aprendizaje a partir de sus conocimientos actuales: qué había aprendido y qué necesitaba mejorar o aprender en el futuro.

Cada herramienta tuvo el comentario de uno de los profesores y de al menos dos compañeros, valorando su diseño, su aplicación y, en su caso, la innovación introducida.

La actividad "Caja de herramientas del profesor" supuso el $20 \%$ de la calificación final de la asignatura. En cada publicación se valoró lo adecuado de la actividad de aprendizaje diseñada para los objetivos propuestos, lo adecuado de la(s) herramienta(s) TIC utilizada y la capacidad de reflexión sobre la utilidad de las actividades desarrolladas para el aprendizaje de las áreas de tecnología e informática en la ESO.

\subsection{Tecnología utilizada}

La "Caja de herramientas del profesor" se construyó en la wiki Sustancia Gris ${ }^{1}$, alojada en wikispaces, administrada y gestionada por una alumna. Sus funciones consistieron en crear la estructura para contener las herramientas creadas por sus compañeros, mantener el índice y resolver las incidencias de páginas mal enlazadas o desenlazadas accidentalmente. El nombre fue elegido por los estudiantes entre los que ellos mismos propusieron. Lamb (2004) afirma que para que la utilización de una wiki proporcione los resultados buscados no debe ser "impuesta desde arriba" sino estructurada y gestionada por los estudiantes.

La elección de una wiki como plataforma se basó en dos razones. Por un lado es una herramienta diseñada expresamente para ser construida de manera colaborativa, que permite tanto la creación y posterior edición de las páginas por cualquiera de los autores como los comentarios de cualquiera de los miembros a cualquier página. Por otro, los estudiantes habían trabajado con una wiki en una asignatura del cuatrimestre anterior, por lo que ya estaban familiarizados con su entorno y su manejo.

1 Alojada en sustanciagris.wikispaces.com

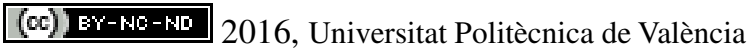

Congreso In-Red (2016) 


\subsection{Evaluación de la experiencia}

El diseño de la experiencia incluye la valoración de la misma por alumnos y profesores.

Los alumnos evaluaron el impacto subjetivo en relación al interés y la aplicabilidad de la caja de herramientas como elemento de aprendizaje y formación profesional mediante un cuestionario Likert "on line", en el que se garantizaba el anonimato. Los items del cuestionario atendían a tres dimensiones: la valoración de la "Caja de herramientas del profesor" como actividad de aprendizaje (relación entre teoría y práctica, cooperación entre compañeros, valoración de los comentarios del profesor y de los compañeros o utilidad para su aprendizaje de cada una de las cuatro secciones de la herramienta), la valoración como herramienta de aprendizaje profesional (desarrollo de habilidades propias de la profesión, la posibilidad de seleccionar la herramienta que mejor se adapta a su estilo personal o formación permanente) y la valoración la estructura y frecuencia de la actividad.

Los profesores evaluaron la calidad de los aprendizajes de los alumnos logrados con la "Caja de herramientas del profesor" en base a los criterios de calificación expresados al final de 3.1 .

\section{Resultados}

El cuestionario fue contestado por 16 estudiantes, lo que representa el $67 \%$ de los 24 alumnos matriculados en la asignatura y el $94 \%$ de los 17 que participaron regularmente en esta actividad.

\subsection{Valoración de los estudiantes}

\subsubsection{Dimensión 1: Caja de herramientas como actividad de aprendizaje}

Los estudiantes valoraron positivamente la "Caja de herramientas del profesor" como actividad de aprendizaje: "al tener que pensar en la actividad concreta, te ayuda a reflexionar sobre la misma, ver los objetivos y los beneficios que se pueden obtener con ella. Hacer el ejercicio de plantear la actividad a veces es complicado de pensar, y es un reto". Les permitió "Conocer herramientas que desconocía y poder actualizarme", "Averiguar formas que desconocía sobre métodos para impartir clase" o "Proponer actividades variadas".

Mediante una escala Likert de seis grados el 68,75\% (11 alumnos) manifestó estar bastante o completamente de acuerdo con que les ayudó a establecer la relación entre la teoría y la práctica de lo que un docente tiene que hacer en el aula $(0=$ En completo desacuerdo; $5=$ Completamente de acuerdo. Med=3,94; SD: 1,12). Solo un estudiante $(6,25 \%)$ manifestó estar en bastante desacuerdo con este ítem y ninguno estuvo en completo desacuerdo.

La apreciación de que la actividad sirvió para desarrollar la cooperación entre los compañeros fue menor. Solo un 37,5\% (6 alumnos) manifestó estar bastante o completamente de acuerdo con este ítem, otro $37,5 \%$ estuvo parcialmente de acuerdo y un 
$12,5 \%$ (2 alumnos) bastante en desacuerdo (Med= 3,13; SD: 1,20). Ninguno estuvo en completo desacuerdo.

El tercer aspecto de esta dimensión midió como valoraron los estudiantes si los comentarios del profesor y de los compañeros a cada una de las herramientas que desarrollaban les ayudaron a mejorar su aprendizaje. Valoraron muy positivamente (Med: 4,06; SD: 1,00) la ayuda que proporcionaban los comentarios del profesor. La valoración a los comentarios de los compañeros fue menor (Med: 3,31; SD: 1,40). El detalle de estas valoraciones se puede ver en la tabla 1 .

Tabla 1. Valoración de los comentarios

\begin{tabular}{ccccccc}
\hline & & \multicolumn{5}{c}{ Han ayudado } \\
Hechos por & Mucho & Bastante & Algo & Poco & Muy poco & Nada \\
\hline El profesor & $43,75 \%$ & $25,0 \%$ & $25,0 \%$ & $6,25 \%$ & 0 & 0 \\
Los compañeros & $12,5 \%$ & $50,0 \%$ & $12,5 \%$ & $12,5 \%$ & $6,25 \%$ & $6,25 \%$ \\
\hline
\end{tabular}

4.1.2. Dimensión 2: Caja de herramientas como herramienta de aprendizaje profesional

Para valorar si los estudiantes consideraban la "Caja de herramientas del profesor" como una actividad apropiada para su aprendizaje profesional se les plantearon tres afirmaciones con una escala Likert de seis grados: $0=$ En completo desacuerdo; $5=$ Completamente de acuerdo.

La afirmación de que elaborar las herramientas les ayudaba a "desarrollar habilidades necesarias para ejercer la profesión docente" obtuvo una valoración media de 3,81 (SD: $0,66)$.

La afirmación de que la "Caja de herramientas del profesor" "será un instrumento a tener en cuenta en su formación permanente como docente" obtuvo una valoración media de 3,88 (SD: 0,81).

Tabla 2. Valoración como herramienta de aprendizaje profesional

\begin{tabular}{ccccccc}
\hline & \multicolumn{3}{c}{ Nivel de acuerdo } & \multicolumn{3}{c}{ Nivel de acuerdo } \\
& Completo & Bastante & Algo & Algo & Bastante & Total \\
\hline $\begin{array}{c}\text { Desarrollar } \\
\text { habilidades }\end{array}$ & $12,50 \%$ & $56,25 \%$ & $31,25 \%$ & 0 & 0 & 0 \\
$\begin{array}{c}\text { Formación } \\
\text { permanente }\end{array}$ & $25,0 \%$ & $37,50 \%$ & $37,50 \%$ & 0 & 0 & 0 \\
$\begin{array}{c}\text { Adaptación } \\
\text { personal }\end{array}$ & $12,50 \%$ & $56,25 \%$ & $18,75 \%$ & $6,25 \%$ & $6,25 \%$ & 0 \\
\hline
\end{tabular}

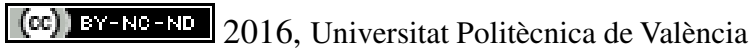

Congreso In-Red (2016) 
La tercera afirmación, que la "Caja de herramientas del profesor" "permite la adaptación de lo trabajado en clase a su manera de aprender y hacer las cosas" obtuvo una valoración media de 3,63 (SD: 1,02).

El detalle de estas valoraciones se puede ver en la tabla 2.

\subsubsection{Dimensión 3: Construcción de la Caja de herramientas}

A los estudiantes se les pidió que valoraran cómo había contribuido cada una de los cuatro aspectos de cada herramienta (descripción de la TIC - descripción de la actividad de aprendizaje - reflexión sobre la adecuación de la actividad y la TIC para lograr los objetivos propuestos - autoevaluación) en su aprendizaje. También se les pidió su valoración sobre la frecuencia requerida.

La sección mejor valorada fue la descripción de la actividad de aprendizaje (Med = 4,19; $\mathrm{SD}=0,54)$. También fueron bien valoradas la reflexión sobre la adecuación para lograr los objetivos (Med = 3,75; SD = 1,0) y la descripción de la TIC utilizada (Med = 3,69; SD = 0,70). La autoevaluación fue el aspecto peor valorado (Med $=3 ; \mathrm{SD}=1,26$ ). La figura 2 muestra un detalle de las valoraciones de cada aspecto.

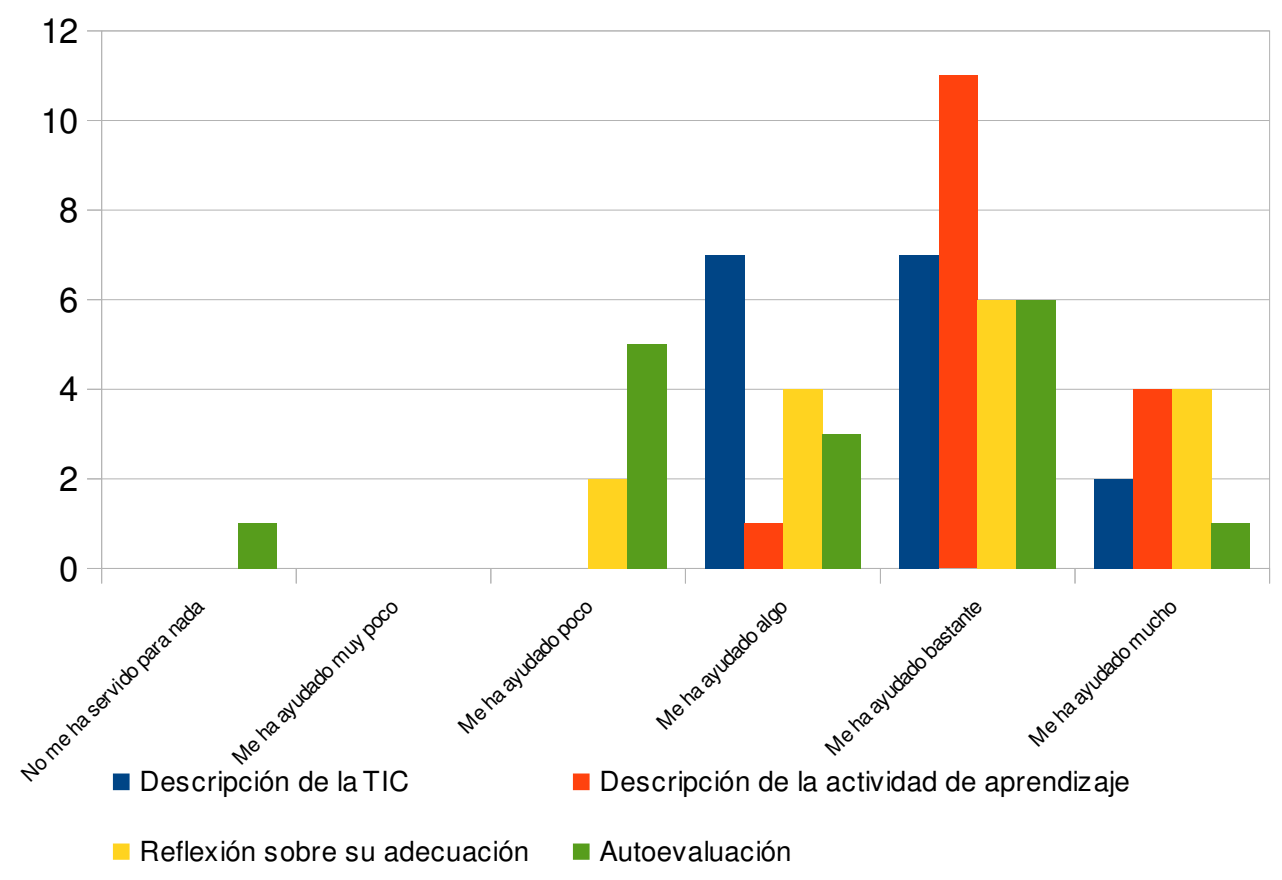

Fig 2: Valoración de las secciones de cada herramienta

Un 62,50\% de los estudiantes (diez) manifestaron no estar de acuerdo con la frecuencia requerida para realizar la actividad (al menos una entrada semanal en el área de informática y una entrada de grupo por cada sesión de trabajo). Ninguno estuvo completamente de 
acuerdo y solo dos $(12,5 \%)$ estuvieron bastante de acuerdo. La valoración media fue de $2,06(\mathrm{SD}=1,18)$.

\subsection{Valoración del profesorado}

El profesorado valoró positivamente el aprendizaje conseguido por los estudiantes al realizar esta actividad. Las herramientas que diseñaban partían de una descripción bien fundamentada de la TIC en la que se basaban, marcaban claramente los objetivos a conseguir, entre los que había tanto objetivos propios de los contenidos del área en cuestión como de desarrollo del pensamiento crítico o de las habilidades necesarias para trabajar en equipo, y tenían un diseño claro. La reflexión que realizaban sobre la adecuación de la TIC y la actividad para conseguir los objetivos propuestos estaba bien argumentada. El cuarto aspecto, la autoevaluación, es el que más les costó realizar. Hubo una evolución. En las primeras entradas en lugar de hacer un ejercicio de autoevaluación - ¿Qué necesito aprender y qué es lo que considero que ya sé hacer? - enumeraban los requerimientos que un docente debería tener en cuenta para realizar la actividad propuesta. A partir de la segunda y la tercera semana, si bien algunos estudiantes siguieron realizando un listado de requerimientos o una autoevaluación en impersonal ("Será necesario diseñar...") un 60\% realizaban una buena autoevaluación: “... uno de los primeros miedos que me planteo como docente...", “... En cuanto a los contenidos estoy bastante verde ...", "Como docente debería dejarles claro ..." o “... no sé como de complicado puede ser ...".

La calificación media de las entradas personales ha sido 2,67 (sobre un máximo de 3; SD = $0,41)$.

\section{Conclusiones}

Con la construcción de la "Caja de herramientas del profesor" se han alcanzado tanto el objetivo de los estudiantes - adquirir las competencias necesarias para el desempeño de la práctica profesional - como el de los profesores - que los estudiantes aprendieran a construir herramientas e instrumentos para enriquecer y mejorar los procesos de enseñanza - aprendizaje de sus futuros alumnos. Además se ha conseguido una caja con herramientas sólidas, a la que podrán recurrir una vez iniciada su práctica profesional.

Esta actividad, además de demostrar que ha sido útil para fomentar competencias transversales como el trabajo colaborativo y el desarrollo del pensamiento crítico, ha propiciado la participación de los estudiantes en un contexto similar al de su práctica profesional, en la que tendrán que programar las unidades de aprendizaje.

El reducido número de alumnos ha supuesto una ventaja y un inconveniente. Ha sido una ventaja porque ha permitido al profesorado realizar un seguimiento personal, comentando semanalmente las entradas de los alumnos que han participado en la actividad, lo que ha proporcionado a profesores y alumnos información relevante sobre el grado de adquisición de los objetivos de aprendizaje de la asignatura.

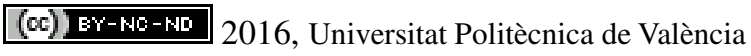

Congreso In-Red (2016) 
No obstante, el reducido número de alumnos ha impedido que la "Caja de herramientas del profesor" tenga una mayor riqueza y variedad de propuestas.

La experiencia aquí descrita se ha circunscrito a una asignatura de una especialidad específica del Máster en profesorado pero, apoyándonos en los resultados positivos obtenidos, opinamos que es transferible a otras especialidades del Máster y también a otras titulaciones y grados universitarios. Construir de manera colaborativa una "Caja de herramientas" propias de un oficio es una actividad de aprendizaje que acercará las aulas a la práctica profesional.

\section{Referencias}

FALCO, J. M. (2014). "El portafolio digital como herramienta de evaluación en el Máster en profesorado" en Alejandre, J.L. Buenas prácticas en la docencia universitaria con apoyo de TIC: experiencias en 2013 (pp. 137-148). Prensas Universitarias de Zaragoza.

Koehler, M. J., y Mishra, P. (2008) Handbook of Technological Pedagogical Content Knowledge (TPCK) for Educators. Routledge

HANSON, M. H., y HeRZ, R. S. (2011). "A ‘toolbox approach’ for developing thoughtfully structured, creative art experiences" en Art Education, v64 n1, pp. 33.

LAMB, B. (2004) "Wide open spaces: Wikis, ready or not" en EDUCAUSE review, v39 n5, pp. 36-49

Perry Marshall (@PerryMarshall) "No one who ever bought a drill wanted a drill. They wanted a hole”. 27 feb 2014, 17:58 [TwITTER]. https://twitter.com/PerryMarshall/status/439082004781350912 [CONSULTA: 21 DE MARZO DE 2016] 